\section{Estadiamento do Carcinoma Diferenciado de Tireóide pela Classificação AJCC/TNM}

D IFERENTES SISTEMAS PROGNÓSTICOS são propostos para o carcinoma diferenciado de tireóide (CDT) baseado nos fatores prognósticos: MACIS (distant metastasis, age, completeness of primary tumor ressection, local invasion, tumor size), AMES (age, distant metastasis, tumor extent, tumor size), AGES (age, grade, tumor extent, tumor size), TNM (tumor, node metastasis, distant metastasis), classificação do EORCT (European Organization for Research on Treatment of Cancer) e a proposta por DeGroot (linfonodo, invasão extra-tireoideana, metástases distantes), entre outros. Todos estes sistemas estão validados na predição de mortalidade relacionada à doença em longo prazo. Assim, aceita-se a utilização de qualquer um destes, não havendo uma clara superioridade de algum e ocorrendo, inclusive, diferenças populacionais (1). A desvantagem do uso de sistemas prognósticos diferentes é que dificulta a aplicação das conclusões de estudos em populações classificadas de forma diferente, e o agrupamento de dados em artigos de revisão, consensos ou meta-análises. Mesmo apresentando limitações $(1,2)$, o sistema de classificação da AJCC (American Joint Committee on Cancer) / TNM é conhecido universalmente para todos os tumores e considera muitos dos fatores prognósticos no CDT. Por estas razões, defendemos que esta classificação seja a padronizada nas publicações sobre $\operatorname{CDT}(1,3,4)$.

A AJCC modificou esta classificação em janeiro de 2003 (5). Para avaliação do novo TNM (tabelas 1 e 2), estudamos retrospectivamente 168 pacientes quanto ao prognóstico, 5 anos após a terapia inicial (tireoidectomia total seguida de radioiodoterapia) (tabela 3). Apesar da pequena série e curto período de seguimento, verificamos o valor desta classificação prognóstica.

Propomos aos pesquisadores do nosso país a adoção do sistema da AJCC/TNM nas publicações, o que facilita a aplicação dos resultados nos outros serviços e permite o agrupamento de dados de diferentes centros, possibilitando uma maior casuística.

\section{cartas ao editor}

Pedro Weslley S. Rosário

Álvaro Luís Barroso

Eduardo Lanza Padrão

Leonardo Lamego Rezende

Saulo Purisch

Serviços de Medicina Nuclear e Endocrinologia da Santa Casa de Belo Horizonte, $M G$. 
Tabela 1. Classificação AJCC/TNM para CDT.

\begin{tabular}{|c|c|c|}
\hline (T) Tumor primário & (N) Metástases linfonodais & (M) Metástases distantes \\
\hline $\begin{array}{l}\text { Tx - não pode ser avaliado } \\
\text { do }\end{array}$ & Nx - não pode ser avaliado & Mx - não pode ser avalia- \\
\hline T1 - 2cm limitado à tireóide & NO - ausente & MO - ausente \\
\hline T2 - > 2 a 4cm limitado à tireóide & $\begin{array}{l}\text { Nla - metástases em linfondos } \\
\text { no nível IV (pré-traqueal, } \\
\text { paratraqueal, pré-laringeo) }\end{array}$ & M1 - presente \\
\hline $\begin{array}{l}\text { T3 - > 4cm ou extensão mínima } \\
\text { para fora da tireóide }\end{array}$ & $\begin{array}{l}\text { N1b - metástases cervical } \\
\text { unilateral, bilateral ou } \\
\text { contralateral ou mediastinal superior }\end{array}$ & \\
\hline \multicolumn{3}{|c|}{ T4a - extensão para tecido subcutâneo, laringe, traquéia, esôfago ou recorrente laríngeo } \\
\hline T4b - invade a fáscia pré-vertebra & nvolvendo carótida ou vasos medic & tinais \\
\hline
\end{tabular}

Tabela 2. Classificação AJCC/TNM para CDT.

\begin{tabular}{|c|c|c|}
\hline Estadiamento & $<45$ anos & $>45$ anos \\
\hline I & Qualquer T, qualquer N, M0 & $\mathrm{Tl}, \mathrm{NO}, \mathrm{MO}$ \\
\hline II & Qualquer T, qualquer N, M1 & T2,NO,MO \\
\hline III & & $\begin{array}{l}\text { T3,NO,MO } \\
\text { Tl-3,Nla,MO }\end{array}$ \\
\hline IV A & & $\begin{array}{c}\text { T4a,qualquer N,M0 } \\
\text { Tl-3,N1b,M0 }\end{array}$ \\
\hline IV B & & T4b,qualquer N,MO \\
\hline IV C & & Qualquer $\mathrm{T}$, qualquer $\mathrm{N}, \mathrm{M} 1$ \\
\hline
\end{tabular}

Tabela 3. Prognóstico do CDT baseado na classificação AJCC-TNM.

\begin{tabular}{|c|c|c|}
\hline Estadiamento AJCC-TNM & Mortalidade * & Pacientes livres de doença \\
\hline$I(n=62)$ & $0 \%$ & $95 \%$ \\
\hline II $(n=36)$ & $0 \%$ & $86 \%$ \\
\hline III $(n=40)$ & $5 \%$ & $50 \%$ \\
\hline$V(n=30)$ & $33 \%$ & $30 \%$ \\
\hline
\end{tabular}

* $p<0,05$

\section{REFERÊNCIAS}

1. Passler C, Prager G, Scheuba C, Kaserer K, Zettinig G, Niederle B. Application of staging systems for differentiated thyroid carcinoma in an endemic goiter region with iodine substitution. Ann Surg 2003,237:227-34.

2. Mazzaferri EL. NCCN thyroid carcinoma practice guidelines. Oncology 1999;13(suppl. 11A):391-442. http://www.ncen.org

3. AACE-AAES medical-surgical guidelines for clinical practice: management of thyroid carcinoma. Endocr Pract 2001;7:202-20.

4. Brierley JD, Panzarella T, Tsang RW, Gospodarowick MK, O'Sullivan B. A comparison of different staging systems predictability of patient outcome: thyroid carcinoma as an example. Cancer 1997;79:2414-23.
5. AJCC Cancer Stating Manual, $6^{\text {th }}$ edition. American Joint Committee on Cancer. http://www.cancerstating.org

Endereço para correspondência:

Pedro Weslley Souza do Rosário

Centro de Estudos e Pesquisa

Clínica de Endocrinologia e Metabologia (CEPCEM)

Av. Francisco Sales $1111,5^{\circ}$ andar, Ala D

30150-221 Belo Horizonte, MG

Fax: (31) 3213-0836

E-mail: pedrorosario@globo.com 